\title{
Energy analysis and experimental evaluation of momentum exchange impact damper with pre- straining spring mechanism
}

Lovely Son , Eka Satria , Jhon Malta \& Berry Yuliandra

To cite this article: Lovely Son , Eka Satria , Jhon Malta \& Berry Yuliandra (2020): Energy analysis and experimental evaluation of momentum exchange impact damper with pre-straining spring mechanism, Australian Journal of Mechanical Engineering, DOI: 10.1080/14484846.2020.1794501

To link to this article: https://doi.org/10.1080/14484846.2020.1794501

曲 Published online: 20 Jul 2020.

6 Submit your article to this journal

Q View related articles $\sqsubset$

View Crossmark data $\nearrow$ 


\title{
Energy analysis and experimental evaluation of momentum exchange impact damper with pre-straining spring mechanism
}

\author{
Lovely Son, Eka Satria, Jhon Malta and Berry Yuliandra \\ Department of Mechanical Engineering, Andalas University, Padang, Indonesia
}

\begin{abstract}
The aim of this research is to evaluate the performance of impact-induced vibration control using momentum exchange impact damper with pre-straining spring mechanism (PSMEID) based on the energy analysis and verifying the effectiveness of the proposed method experimentally using a simple three-body collision model. It is shown from the simulation study that the fraction of energy absorbed by the primary mass was small if the excitation frequency differs from the primary mass natural frequency. In the case of excitation frequency is lower than the primary mass natural frequency, most of the impact energy is transferred to the damper mass. For excitation frequencies higher than the primary mass natural frequency, a large amount of impact energy is reflected after the collision. The energy absorption ratio is small if the damper mass ratio $\left(M_{d} / M_{p}\right)$ approaches 0.2 and the ratio between pin force $\left(f_{\text {pin }}\right)$ and the reference friction force $\left(f_{\text {fref }}\right)$ closest to 1 . The optimum condition of the pre-straining spring stiffness increases when the impact damper mass ratio is increased. The experiment was conducted to validate the simulation results. The experimental data has shown that the minimum acceleration response is obtained using the pre-straining spring deflection ratio $\left(x_{p s} / x_{\text {psref }}=0.18\right)$.
\end{abstract}

ARTICLE HISTORY

Received 2 March 2020

Accepted 3 July 2020

\section{KEYWORDS}

Impact; damper; vibration; control; PSMEID

\section{Introduction}

Shock-induced vibration problems have attracted the attention of researchers in various research disciplines. The reason is that the impact phenomenon occurs very often in many modern technological devices such as heat exchanger(Goyda and Teh 1989), manufacturing system(Tung and Shaw 1998; Raju and Annesh 2017), vehicle(Bovenzi et al. 2006; Rantaharju et al. 2015) and building floor(Hanagan, Murray, and Premaratne 2003; Son et al. 2007). Some shock vibration phenomena are destructive and should be eliminated. The most important thing to consider in the shock vibration problem is the large acceleration response due to the very high impulsive force acting on the system. This condition can reduce system safety and comfort(Sezgin and Arslan 2012). Furthermore, in some cases, the large amplitude impulsive forces can damage the main system components(Son, Bur, and Rusli 2018).

Some researchers have proposed several methods to minimise the effect of vibration on the system. Increasing the system damping is the most popular technique to dissipate vibration energy(Constantinou and Symans 1993; Goel 2005; Dutta 2020). Even though this technique can reduce the steady-state response amplitude under harmonic excitation, however, it is failing to reduce a large response overshoot that occurs due to the impact load (Lee et al. 2009).
(Hanagan, Murray, and Premaratne 2003) proposed a method using active vibration control to reduce the acceleration and displacement response of a rigid body under shock load excitation. The main drawback of using the active control technique is it needs some sensors and actuators to get a good vibration control performance. (Faraj and Graczykowski 2019) investigated a technique for impact vibration mitigation using fluid-based shock-absorbers. (Yang and Wang 2019) proposed a technique for impact vibration attenuation of beam structure using momentum exchange principle.

A simple method for shock vibration control of one DOF vibration system using the passive momentum exchange damper (PMEID) has been studied by (Son, Matsuhisa, and Utsuno 2008). The passive momentum exchange impact damper not only reduces the steadystate response amplitude but also decreases the maximum response overshoot that occurs at the instant after the impact. Shock vibration control using PMEID is shown schematically in Figure 1(a). $m_{a}, m_{b}$, and $m_{c}$ represent the excitation mass, primary mass, and damper mass, respectively. The primary mass is attached to the free end of the cantilever beam. The primary mass and the damper mass are initially in contact with each other. Contact condition between the primary mass and the damper mass is described using a contact spring. When $m_{a}$ collides with $m_{b}$, some impact energy is transferred from the primary mass to 


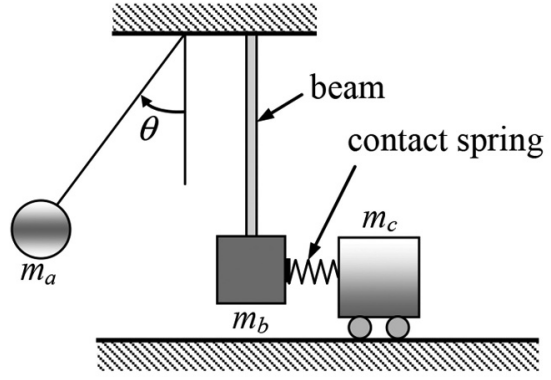

(a)

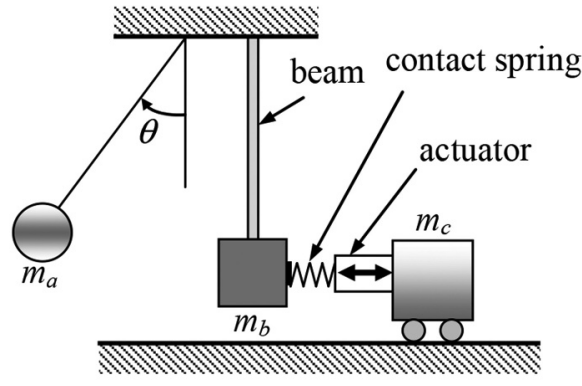

(b)

Figure 1. Schematic diagram of momentum exchange impact damper (a) PMEID (b) AMEID.

the damper mass. As a result, the impact-induced vibration of the primary mass is reduced. For a small impact period, PMEID can effectively reduce the shock vibration response of the primary mass. However, the performance decreases when the impact period is large.

(Son et al. 2010) developed the active momentum exchange impact damper(AMEID) to improve the PMEID performance in reducing the shock vibration response of a mechanical system. Figure 1(b) shows a schematic diagram of shock vibration control using AMEID. Different from PMEID, AMEID utilising an actuator located between the primary mass and the damper mass. When the impact force applied to the primary mass, AMEID simultaneously works and resists the impulsive load on the primary mass by generating a counterbalancing force on the actuator. Even though this technique can effectively reduce the impact-induced vibration response of the primary mass, but its performance decreases when the impact time very short due to a delay in the actuator control action. Furthermore, this method needs a high power actuator to generate a large impulsive force.

This study proposes an alternative method for increasing the performance of momentum exchange impact damper using the pre-straining spring mechanism. This technique is called pre-straining spring momentum exchange impact damper(PSMEID). PSMEID has good performance over a wide range of excitation frequencies, whilst the PMEID performance is only effective at the excitation frequency much larger than the primary mass natural frequency(Son, Bur, and Rusli 2017). Recently, the PSMEID method has been proposed to reduce the impact-induced vibration of UAV landing gear; however, the energy and momentum exchange during impact is still not clearly understood, and the simulation results are not yet experimentally verified (Son, Bur, and Rusli 2018).

In this study, the vibration response attenuation of the impact vibration system using PSMEID was evaluated using the energy exchange analysis. The impact vibration system is modelled as a three-body collision model. The energy analysis is conducted by evaluating the energy reflected by the excitation mass, the energy absorbed by the primary mass, and the energy transferred to the damper mass. The experimental study using a three-body collision model is conducted to validate the results calculated by the simulation.

An important strength of this study is its quantitatively explore the energy and momentum exchange during impact by energy analysis and investigate the effect of PSMEID on the energy exchange between the excitation mass, the primary mass and the damper mass, whilst Son et al. (Son, Bur, and Rusli 2017) had focused on the response analysis of a vibration system using PSMEID. Additionally, an important contribution of this study is that the experimental results had shown the feasibility of the proposed method to be applied in the real condition. Furthermore, the results of this study open a new avenue for future research on impact vibration control using the momentum exchange principle.

\section{Three body collision model with PSMEID}

Figure 2 shows a three-body collision model with PSMEID. The excitation mass $M_{b}$ moves with a constant velocity $v_{b}^{-}$towards the primary mass $M_{p}$ that is initially contacting with PSMEID. PSMEID consists of two masses, namely the damper mass $M_{d}$ and the contact mass $M_{c}$. A pre-straining spring $k_{p s}$ is placed between $M_{c}$ and $M_{d}$. The pre-straining spring is initially compressed by a certain compressive force $\left(f_{t}\right)$ . The primary mass is connected with PSMEID through a contact spring $k_{c}$. At the instant, after the excitation mass $M_{b}$ collides the primary mass, the prestraining spring releases, and a reaction force works to counterbalance the impact force on the primary mass.

The equations of motion for the three-body collision model with PSMEID as shown in Figure 2 are written as follows:

$$
\begin{gathered}
M_{b} \ddot{x}_{b}+f_{b}=0 \\
M_{p} \ddot{x}_{p}+k_{p} x_{p}+f_{c}-f_{b}=0
\end{gathered}
$$




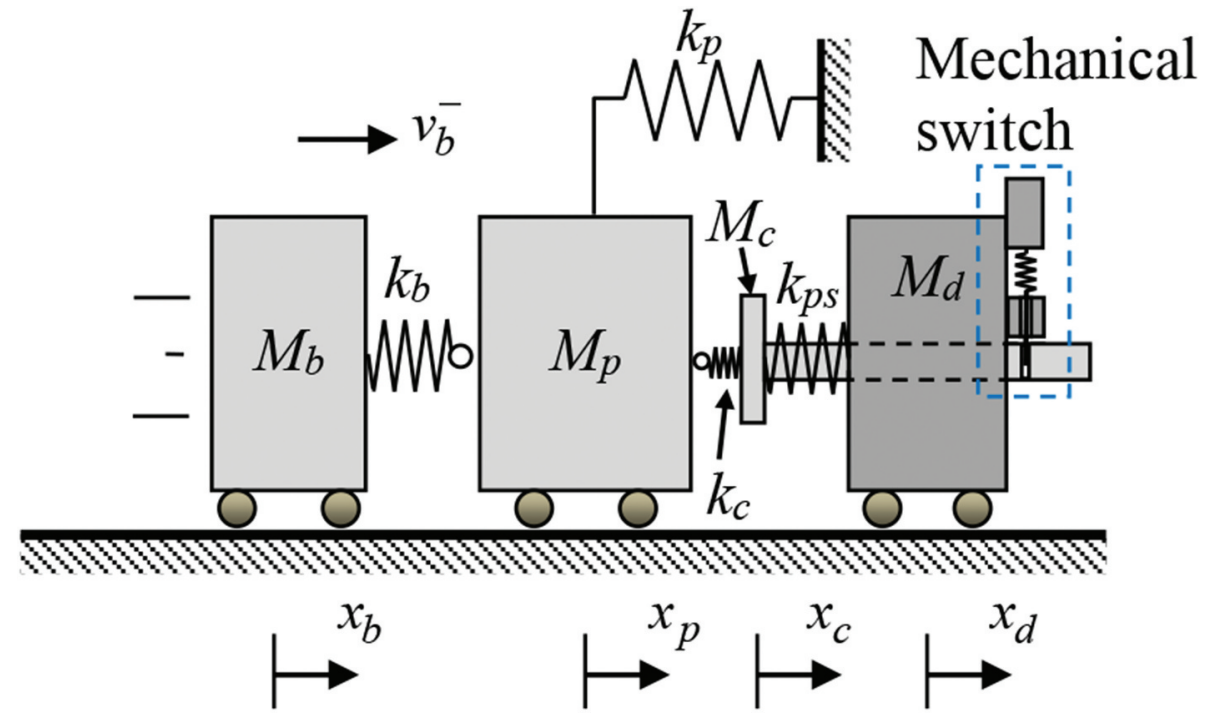

Figure 2. Three-body collision model with PSMEID.

$$
\begin{gathered}
M_{c} \ddot{x}_{c}+f_{p s}-f_{c}-f_{t}=0 \\
M_{d} \ddot{x}_{d}-f_{p s}+f_{t}=0
\end{gathered}
$$

The excitation force $f_{b}$ in Eq.(1) can be written as follows:

$$
f_{b}= \begin{cases}k_{b}\left(x_{b}-x_{p}\right), & \text { if } x_{b} \geq x_{p} \\ 0, & \text { if } x_{b}<x_{p}\end{cases}
$$

The initial conditions for mass $m_{b}$ as shown in Figure 2 are $x_{b 0}=0$ and $v_{b 0}=v_{b}^{-}$. The contact force $f_{c}$ is given by:

$$
f_{c}= \begin{cases}k_{c}\left(x_{p}-x_{c}\right), & \text { if } x_{p} \geq x_{c} \\ 0, & \text { if } x_{p}<x_{c}\end{cases}
$$

$f_{p s}$ is the reaction force of the pre-straining spring due to its initial deflection and the relative displacement between $x_{c}$ and $x_{d}$ as given by:

$$
f_{p s}=k_{p s}\left(x_{p s}+x_{c}-x_{d}\right)
$$

$f_{t}$ is the initial compressing force applied to balance the pre-straining force when the mechanical switch is turned off. When the primary mass receives an impact load, the force for releasing the switch becomes larger than its friction force, thus change the switch status to on. When the switch is turned on, $f_{t}$ will disappear, and the pre-straining spring is released. This condition can be expressed mathematically as:

$$
f_{t}= \begin{cases}k_{p s} x_{p s}, & \text { if } f_{p i n}-f_{f} \leq 0 \\ 0, & \text { if } f_{p i n}-f_{f}>0\end{cases}
$$

$f_{\text {pin }}$ and $f_{f}$ in Eq.(8) is the releasing and friction force acting on the switch mechanism. The friction force is calculated as:

$$
f_{f}=\mu \cdot k_{p s}\left(x_{p s}-x_{c}+x_{d}\right)
$$

\section{Energy analysis}

The energy analysis is conducted to evaluate the energy exchange during the collision of the threebody system with PSMEID as shown in Figure 2. The energy components involved during the energy exchange are described bellows:

(1) The kinetic energy of the excitation mass

$$
K E M_{b}=\frac{1}{2} M_{b} \dot{x}_{b}^{2}
$$

(1) The kinetic energy of the primary mass

$$
K E M_{p}=\frac{1}{2} M_{p} \dot{x}_{p}^{2}
$$

(1) The kinetic energy of the contact mass

$$
K E M_{c}=\frac{1}{2} M_{c} \dot{x}_{c}^{2}
$$

(1) The kinetic energy of the damper mass

$$
K E M_{d}=\frac{1}{2} M_{d} \dot{x}_{d}^{2}
$$

(1) The potential energy of the excitation spring

$P E k_{b}=-\frac{1}{4} k_{b}\left(\left(x_{p}-x_{b}\right)\left|x_{p}-x_{b}\right|-\left(x_{p}-x_{b}\right)^{2}\right)$

(1) The potential energy of the main spring

$$
P E k_{p}=\frac{1}{2} k_{p} x_{p}^{2}
$$

(1) The potential energy of the contact spring

$$
P E k_{c}=-\frac{1}{4} k_{c}\left(\left(x_{c}-x_{p}\right)\left|x_{c}-x_{p}\right|-\left(x_{c}-x_{p}\right)^{2}\right)
$$

(1) The potential energy of the pre-straining spring 


$$
P E k_{p s}=\frac{1}{2} k_{p s}\left(x_{d}-x_{c}-x_{p s}\right)^{2}
$$

The energy absorption ratio is utilised to observe the effect of PSMEID in reducing the primary system energy. The energy absorption ratio is the ratio between the primary system energy after the collision to the initial energy of the excitation mass $\left(E_{p}^{+} / E_{b}^{-}\right)$. The energy absorption ratio describes the portion of energy absorbed by the primary system after the collision. The primary system energy is the sum of the kinetic energy of $M_{p}$ and the potential energy of $k_{p}$ as given by:

$$
E_{p}=K E M_{p}+P E k_{p}=\frac{1}{2} M_{p} \dot{x}_{p}^{2}+\frac{1}{2} k_{b} x_{p}^{2}
$$

The initial energy of the excitation mass is written as follows:

$$
E_{b}^{-}=\frac{1}{2} M_{b}\left(v_{b}^{-}\right)^{2}
$$

\section{Results and discussions}

\subsection{Energy exchange simulation and energy absorption ratio}

The energy fraction involved during the collision is numerically simulated using MATLAB software. The energy fraction is defined as the ratio between the observed energy and the initial energy of the excitation mass. To simulate the shock condition, the excitation mass $M_{b}$ initially collides with the primary mass $M_{p}$ with initial velocity $v_{b}^{-}=1.7 \mathrm{~m} / \mathrm{s}$. Simulation is conducted using the nominal simulation parameters, as depicted in Table 1. The PSMEID nominal parameters are selected based on the authors' previous research (Son, Bur, and Rusli 2017). According to the results obtained from the previous study, the maximum acceleration response of the primary mass was not much affected by the damper and the contact mass variation. The excitation mass contact stiffness and the main system spring stiffness are selected that the main

Table 1. Nominal simulation parameters.

\begin{tabular}{llll}
\hline No & \multicolumn{1}{c}{ Parameters } & \multicolumn{1}{c}{ Value } & \multicolumn{1}{c}{ Unit } \\
\hline 1 & Excitation mass, $M_{b}$ & 1 & $\mathrm{~kg}$ \\
2 & Primary mass, $M_{p}$ & 4.46 & $\mathrm{~kg}$ \\
3 & Damper mass, $M_{d}$ & 0.67 & $\mathrm{~kg}$ \\
4 & Contact mass, $M_{c}$ & 0.157 & $\mathrm{~kg}$ \\
5 & Excitation mass contact stiffness, $k_{b}$ & $1 \times 10^{5}$ & $\mathrm{~N} / \mathrm{m}$ \\
4 & Main system spring stiffness, $k_{p}$ & $1.5 \times 10^{3}$ & $\mathrm{~N} / \mathrm{m}$ \\
5 & PSMEID contact stiffness, $k_{c}$ & $1 \times 10^{6}$ & $\mathrm{~N} / \mathrm{m}$ \\
6 & Pre-straining spring stiffness, $k_{p s}$ & $4.3 \times 10^{3}$ & $\mathrm{~N} / \mathrm{m}$ \\
7 & PSMEID contact damping, $c_{c}$ & 500 & $\mathrm{~N} . \mathrm{s} / \mathrm{m}$ \\
8 & Pre-straining spring initial displacement, $x_{\text {ps }}$ & 0.045 & $\mathrm{~m}$ \\
9 & Spring constant of a mechanical switch, $k_{\text {pin }}$ & $2.24 \times 10^{3}$ & $\mathrm{~N} / \mathrm{m}$ \\
9 & Spring force of mechanical switch, $f_{\text {pin }}$ & 19.5 & $\mathrm{~N}$ \\
10 & Contact time, $T_{c}$ & $9.4 \times 10^{-3}$ & $\mathrm{sec}$ \\
11 & Coefficient of friction, $\mu$ & 0.1 & - \\
12 & Excitation force amplitude, $F_{b}$ & 500 & $\mathrm{~N}$ \\
\hline
\end{tabular}

system natural frequency is much smaller than the excitation frequency. Base on the authors' previous study, PSMEID contact stiffness should be large enough; however, it should not be very large to reduce the high-frequency response. The nominal values of pre-straining spring stiffness and initial displacement are calculated using relation $x_{p s}=0.96 F_{b} / k_{p s}$, where $F_{b}$ is the excitation force amplitude.

Figure 3 shows energy fraction time history of the three-body system using PSMEID during collision period. As shown in Figure 3, the initial energy at $t=0 \mathrm{~s}$ consists of the kinetic energy of $M_{b}\left(K E M_{b}\right)$ and the potential energy of the pre-straining spring $\left(P E k_{p s}\right)$. During the collision period between $M_{b}$ and $M_{p}$, the kinetic energy of $M_{b}$ decreases, and the potential energy of $k_{b}\left(P E k_{b}\right)$ increases. At this time period, a part of energy and momentum from $M_{b}$ are transferred to $M_{d}$. Furthermore, Figure 3 shows that almost half of the kinetic energy of $M_{b}$ is reflected after the collision. The pre-straining spring is released after $t=2 \times 10^{-3} \mathrm{~s}$. As a result, there is an increase in momentum and energy transferred to the damper mass $\left(K E M_{d}\right)$. The maximum value of $K E M_{d}$ occurs at the instant before $M_{d}$ loses contact with the $M_{p}$ $(t=0.02 \mathrm{~s})$.

Contact duration between the primary $\operatorname{mass}\left(M_{p}\right)$ and the damper $\operatorname{mass}\left(M_{d}\right)$ is one of the critical PSMEID characteristic. In the authors' previous research(Son, Bur, and Rusli 2017), the contact duration is controlled by adjusting the gap distance between $M_{p}$ and $M_{d}$. In the current research, the contact duration is tuned using the mechanical switch. Therefore, the pin releasing force $\left(f_{\text {pin }}\right)$ on the mechanical switch is a significant parameter in designing PSMEID. Other important parameters in designing PSMEID are: the excitation frequency $\left(\omega_{b}\right)$, damper mass $\left(M_{d}\right)$ and the pre-straining spring stiffness $\left(k_{p s}\right)$ and initial deflection $\left(x_{p s}\right)$. Evaluation of these parameters variation to the energy absorption ratio is depicted in Figure 4(a-f). Figure 4(a) represents a three-dimensional plot of the energy absorption ratio with a variation of $f_{\text {pin }}$ and $k_{c}$. The reference friction force in Figure 4(a) is calculated as:

$$
f_{\text {fref }}=\mu k_{p s} x_{p s}
$$

$k_{\text {cref }}$ in Figure $4(a)$ is the nominal contact stiffness, as given in Table 1 , and $\mu$ is the friction coefficient between the pin and the primary mass. Figure 4(a) shows that the minimum energy absorption ratio occurs when the value of $f_{\text {pin }}$ near to $f_{\text {fref }}$. Furthermore, it is shown from Figure 4(a) that the effect of contact stiffness to the energy absorption ratio is small.

In the simulation study, the excitation force $\left(f_{b}\right)$ that occurs due to a collision between $M_{b}$ and $M_{p}$ is assumed to be in the form of a half-sine curve with 


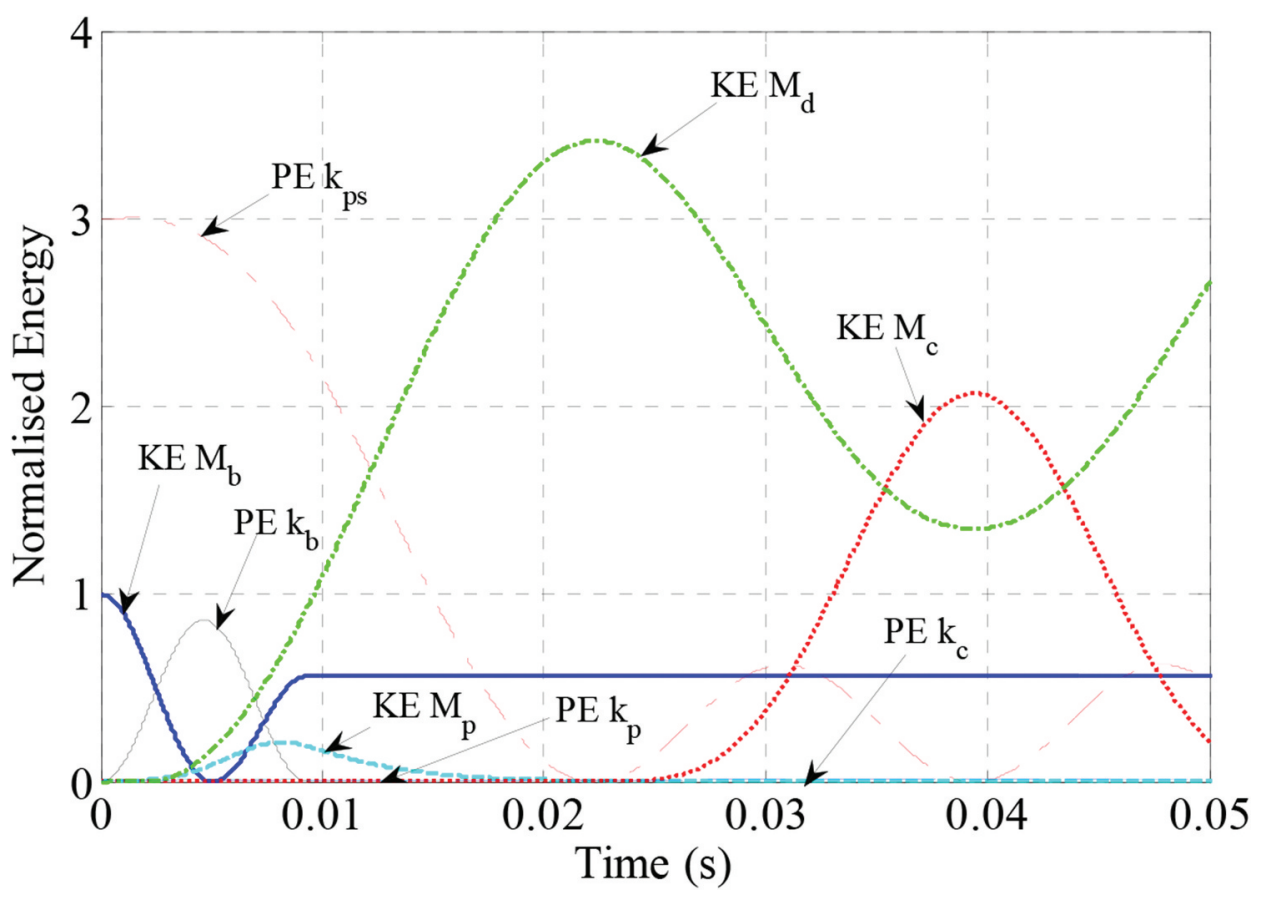

Figure 3. Energy fraction during the collision.

the excitation frequency $\omega_{\mathrm{b}}$. The excitation frequency is calculated as follows:

$$
\omega_{b}=\sqrt{\frac{k_{b}}{M_{b}}}
$$

A three-dimensional plot of the energy absorption ratio versus $f_{\text {pin }}$ and the excitation frequency variation is shown in Figure 4(b). The reference value of the excitation frequency $\omega_{\text {bref }}$ is calculated from Eq.(21) using the nominal parameters of $k_{b}$ and $M_{b}$, as depicted in Table 1. It can be seen from Figure 4(b) that the energy absorption ratio is larger when the excitation frequency increases. This condition is caused by increasing the impulsive force amplitude when the large excitation frequency is used.

The relation of energy absorption ratio to the contact mass and damper mass is shown in Figure 4(c). It can be observed from Figure 4(c) that the energy absorption ratio decreases when the contact mass $\left(M_{c}\right)$ increases. The minimum condition of the energy absorption ratio is obtained when the damper mass ratio $M_{d} / M_{p}$ approaches 0.2 .

The effect of the stiffness and the initial deflection of pre-straining spring on the energy absorption ratio is shown in Figure 4(d). As shown in Figure 4(d), for small deflection, the energy absorption ratio is independent of the pre-straining spring stiffness. Furthermore, for large deflection, the energy absorption ratio increases as the pre-straining spring stiffness are increased.

Figure 4(e) shows the effect of variations in $k_{c}$ and $k_{p s}$ on the energy absorption ratio. In this simulation, $k_{p s r e f}$ is the nominal stiffness of the pre-straining spring, as given in Table 1. The initial deflection of the pre-straining spring is chosen by $x_{p s}=0.46 x_{p s r e f}$. As depicted in Figure 4(e), the energy absorption ratio is small and almost constant when $k_{p s}>0.5 k_{p s r e f}$.

The relation of energy absorption ratio to the primary mass natural frequency $\left(\omega_{p}\right)$ and the contact frequency $\left(\omega_{\mathrm{c}}\right)$ is shown in Figure 4(f). In the simulation, $\omega_{p}$ and $\omega_{c}$ are normalised to the excitation frequency $\left(\omega_{b}\right) . \omega_{p}$ and $\omega_{c}$ are calculated as follows:

$$
\begin{aligned}
& \omega_{p}=\sqrt{\frac{k_{p}}{M_{p}}} \\
& \omega_{c}=\sqrt{\frac{k_{c}}{M_{c}}}
\end{aligned}
$$

It is shown from Figure 4(f) that the energy absorption ratio becomes large if the value of $\omega_{p}$ closest to $\omega_{\mathrm{b}}$. This condition may be due to the resonance.

\subsection{Optimum condition of PSMEID}

The impact problem using PSMEID, as depicted in Figure 2, can be regarded as a collision between oscillators(Pinnington, 2003). In this collision problems, the contact duration occurred for low impact strength is given by:

$$
T_{c}=\frac{\pi \beta}{\omega_{r r} \sqrt{2}}
$$

Where $\omega_{r r}$ is the interaction frequency, and $\beta$ is the impact strength, respectively. For collision between 


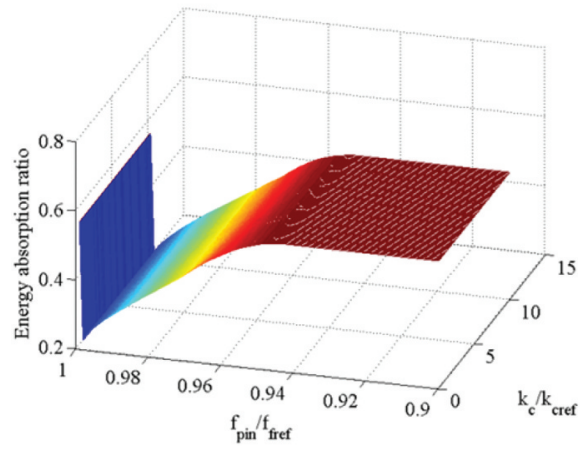

a. Energy absorption ratio vs. $f_{\text {pin }}$ and $k_{c}$

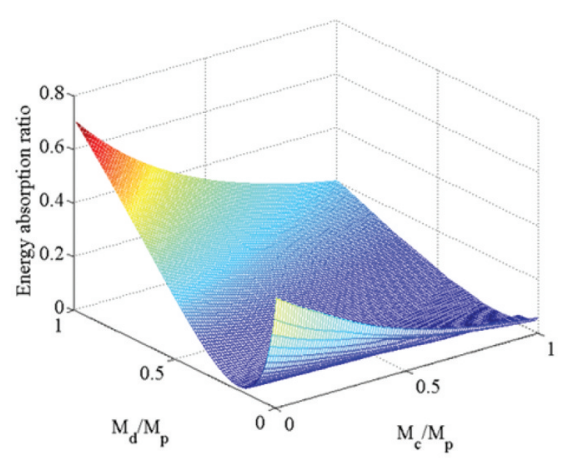

c. Energy absorption ratio vs. $M_{c}$ and $M_{d}$

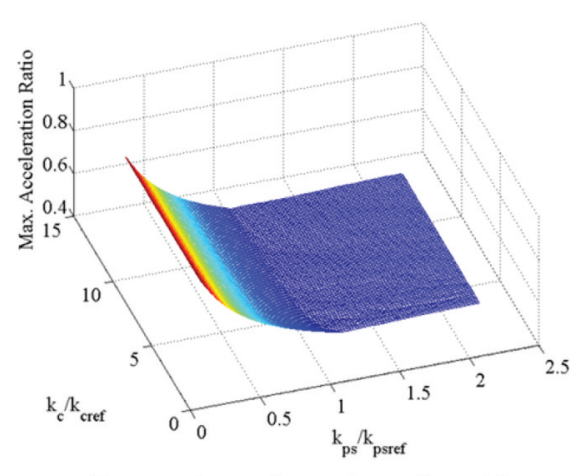

e. Energy absorption ratio vs. $k_{c}$ and $k_{p s}$

Figure 4. Energy absorption ratio variations.

$M_{p}$ and $M_{d}$, the interaction frequency is formulated as follows:

$$
\omega_{r r}=\sqrt{\frac{k_{0}+k_{p s}}{M_{0}}}
$$

$M_{0}$ and $k_{0}$ in Eq.(25) are the interaction mass and stiffness, respectively. For a small damping value, $M_{0}$ and $k_{0}$ can be expressed by:

$$
M_{0}=\frac{M_{p} \cdot M_{d}}{M_{p}+M_{d}} \cdot \text { and } \cdot k_{0}=\frac{k_{p} \cdot k_{d}}{k_{p}+k_{d}}
$$

If the excitation force is assumed as a half sinusoidal curve with frequency $\omega_{b}$ thus the optimum condition for transferring the momentum from the primary mass to the damper mass occurs at the condition (Son et al., 2017):

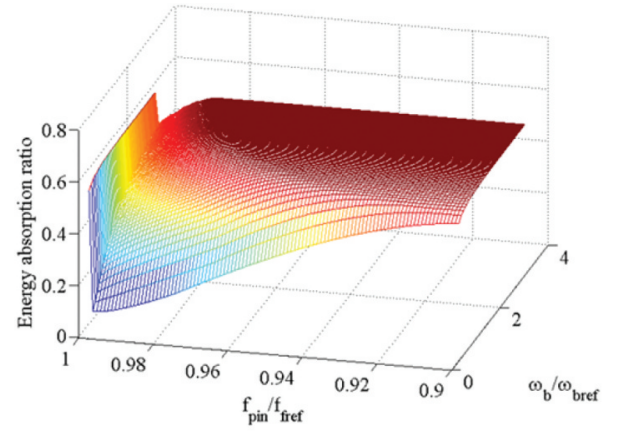

b. Energy absorption vs. $f_{\text {pin }}$ and $\omega_{b}$

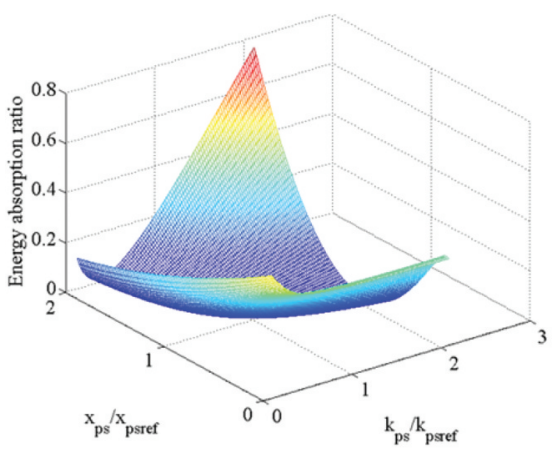

d. Energy absorption ratio vs. $x_{p s}$ and $k_{p s}$

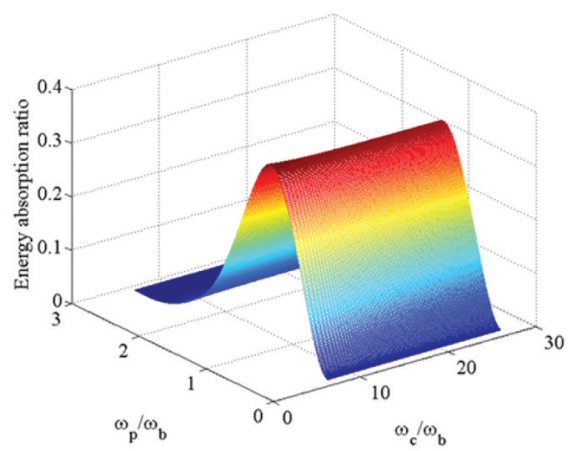

f. Energy absorption ratio vs. $\omega_{p}$ and $\omega_{c}$

$$
T_{c}=\frac{\pi}{\omega_{b}}=\pi \sqrt{\frac{M_{b}}{k_{b}}}
$$

The optimum value of the pre-straining spring stiffness is calculated by combining of Eq.(24) to Eq.(27) as follows:

$$
k_{p s}=\frac{1}{2} \frac{M_{0}}{M_{b}} \beta^{2} k_{b}-k_{0}
$$

In the case of $k_{d}=0$ then $k_{0}=0$, therefore Eq.(28) can be simplified as:

$$
k_{p s}=\frac{1}{2} \frac{M_{0}}{M_{b}} \beta^{2} k_{b}
$$

At the optimum condition, the relationship between the pre-straining spring force and the excitation force is given by (Son et al., 2017):

$$
k_{p s} x_{p s}=0.96 F_{b}
$$




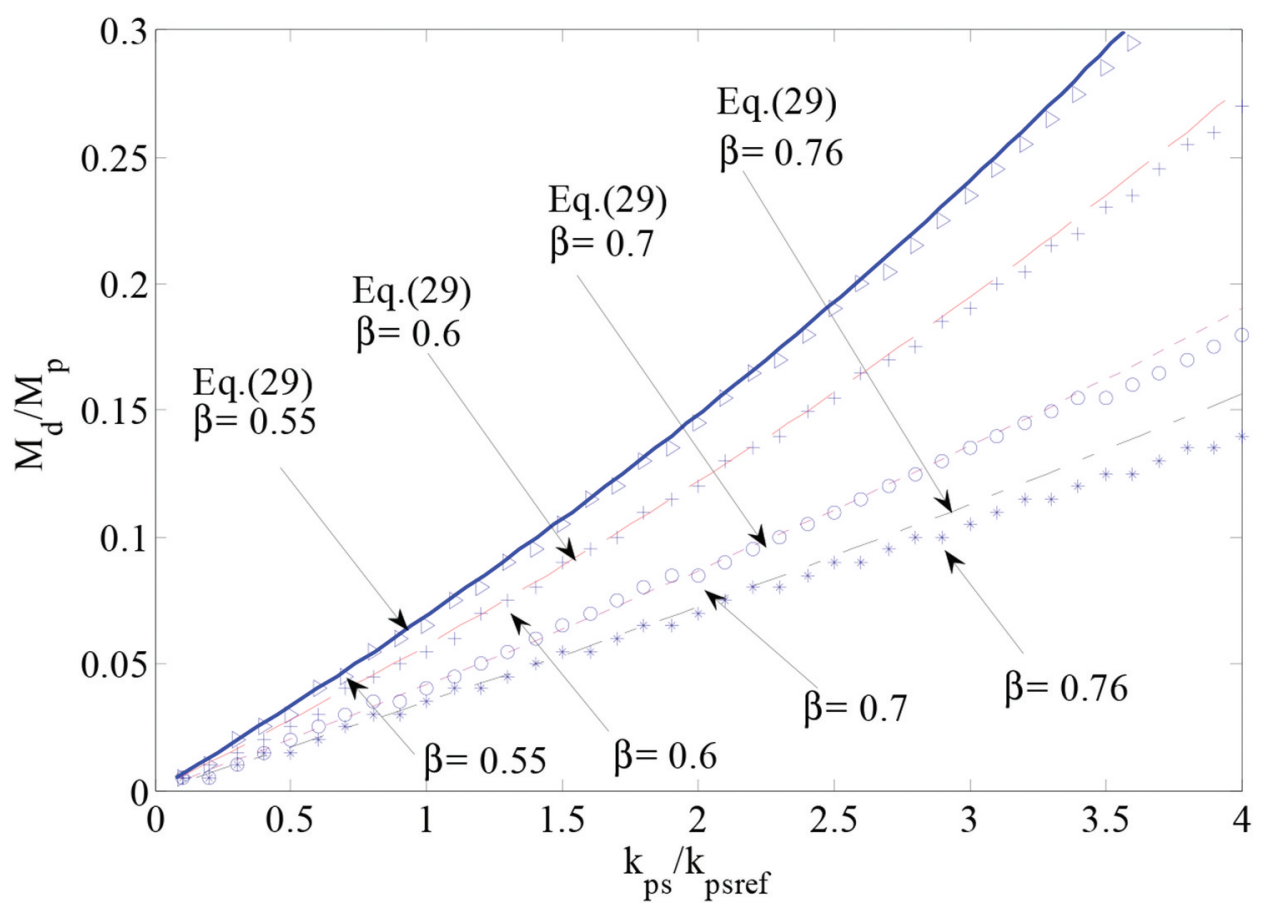

Figure 5. Relationship between mass ratio and $k_{p s}$ at the minimum energy absorption ratio.

Figure 5 shows the relationship between the optimum pre-straining spring stiffness $\left(k_{p s}\right)$ and the damper mass ratio $\left(M_{d} / M_{p}\right)$ obtained from the numerical simulation and analytical calculation. The numerical simulation results in Figure 5 are obtained by simulating Eqs. (1) to (4), and the analytical results are calculated using Eq.(29). It can be observed from Figure 5 that the optimum condition of the pre-straining spring stiffness increases when the mass ratio is increased.

\subsection{Comparison of PMEID and PSMEID performance}

For comparative study, simulation is carried out for the collision of the three-body system using PMEID as shown in Figure 6. The equations of motion of the system with PMEID, as shown in Figure 6 are given by:

$$
M_{b} \ddot{x}_{b}+f_{b}=0
$$

$$
M_{p} \ddot{x}_{p}+k_{p} x_{p}+f_{c}-f_{b}=0
$$

$$
M_{d} \ddot{x}_{d}-f_{c}=0
$$

$$
\begin{aligned}
& f_{b}=\left\{\begin{array}{cc}
k_{b}\left(x_{b}-x_{p}\right), & \text { if } x_{b} \geq x_{p} \\
0, & \text { if } x_{b}<x_{p}
\end{array}\right. \\
& f_{c}=\left\{\begin{array}{cc}
k_{c}\left(x_{p}-x_{d}\right), & \text { if } x_{p} \geq x_{d} \\
0, & \text { if } x_{p}<x_{d}
\end{array}\right.
\end{aligned}
$$

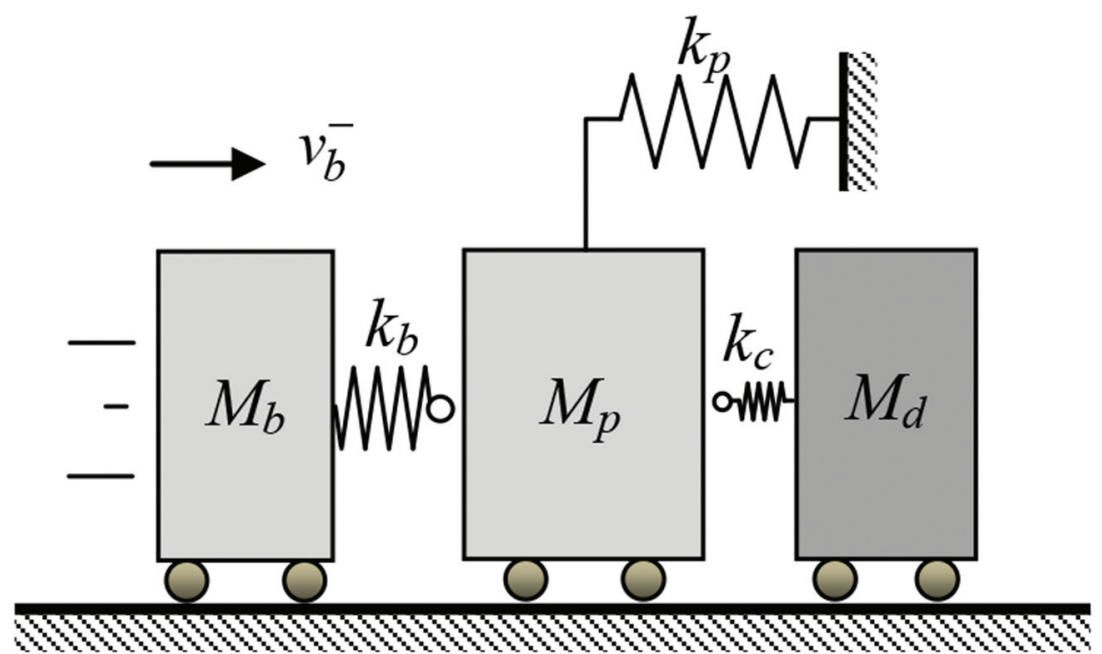

Figure 6. Thre body collision model with PMEID. 
The comparison of PSMEID and PMEID performance in reducing the energy absorption ratio of the primary mass due to shock load with variations of the primary mass natural frequency is depicted in Figure 7 (a). As shown in Figure 7(a), PSMEID is better than PMEID in reducing the energy absorption ratio of the primary mass. For $\omega_{p} / \omega_{b}$ closest to 1 , the energy absorption ratio using PSMEID increases due to resonance.

The normalised energy distribution during the impact period for the low primary mass natural frequency (point A) is shown in Figure 7(b). As depicted in Figure $7(b)$, the reflected energy is 0.55 , and the energy transferred to the damper mass is 3.3 times the initial energy of $M_{b}$

Figure 7(c) shows the energy fraction distribution when the natural frequency of the primary mass closest to the excitation frequency (point B). The reflected energy is almost the same as that obtained using low natural frequency (point A). However, the energy ratio transferred to the damper mass is less than 3. As a result, more energy is absorbed by the primary system $\left(\mathrm{KE} M_{p}+\mathrm{PE} k_{p}\right)$.

Figure $7(\mathrm{~d})$ shows the energy fraction distribution when the natural frequency of the primary mass is larger than the excitation frequency (point C). It can be seen from Figure 7 (d) that almost all energy from the excitation mass is reflected after the collision. The energy transferred to the damper mass is almost the same as the initial energy of the pre-straining spring. The absorbed energy by primary mass is less than that obtained from the previous case (point $\mathrm{B}$ ).

Figure $8(\mathrm{a}-\mathrm{d})$ shows the effect of damper mass variation on the performance of PSMEID and PMEID in reducing the energy absorption ratio of the primary mass. The damper mass $M_{d}$ are varied from $0.01 M_{p}$ to $M_{p}$, as shown in Figure $8(\mathrm{a})$. The contact mass and stiffness of PSMEID are chosen equal to those nominal parameter values, as shown in Table 1. As shown in Figure 8(a), PSMEID has a better performance than PMEID in reducing the energy absorption ratio when $M_{d}$ is less than $0.5 M_{p}$. The optimum condition for PSMEID performance occurs when $M_{d}=0.2 M_{p}$.

Figure $8(\mathrm{~b})$ shows the energy fraction distribution at point $P$. It is shown from Figure 8 (b) that $55 \%$ of the excitation mass initial energy is reflected. After the collision, some energy is transferred to the damper mass and the contact mass. The total energy transferred to $M_{d}$ and $M_{c}$ is 3.3 times the initial energy of $M_{b}$.

Figure 8(c) shows the energy fraction distribution at point $Q$. Based on the results depicted in Figure 8(c), $55 \%$ of the excitation mass initial kinetic energy is reflected. The total energy of $M_{c}$ and $M_{d}$ after the collision is 3.4 times the initial energy. The total energy transferred to the damper mass is higher than that obtained from point $P$.

The energy fraction distribution at point $R$ is shown in Figure 8(d). In this case, the energy transferred to the damper mass is less than three times of the initial

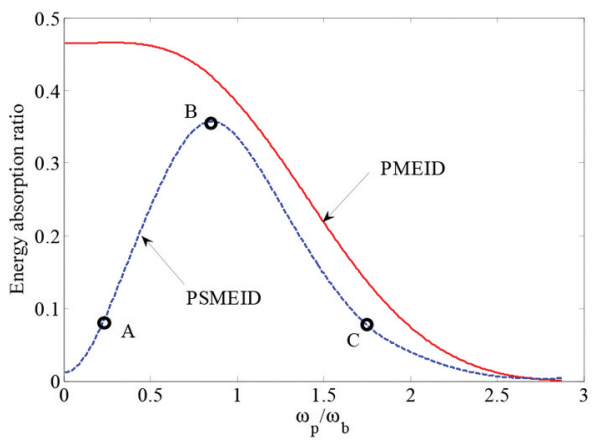

a. Energy absorption ratio vs. $\omega_{p} / \omega_{b}$

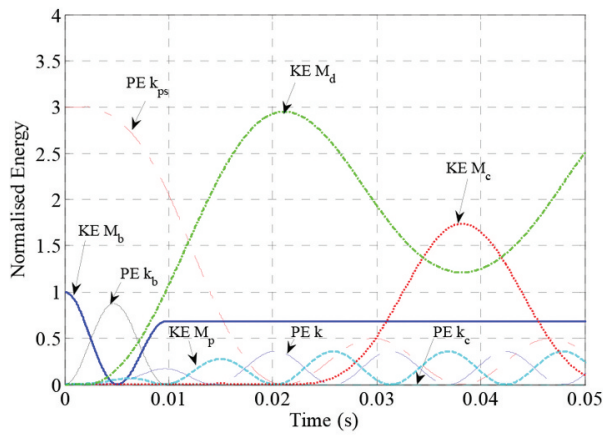

c. Energy fraction at point B

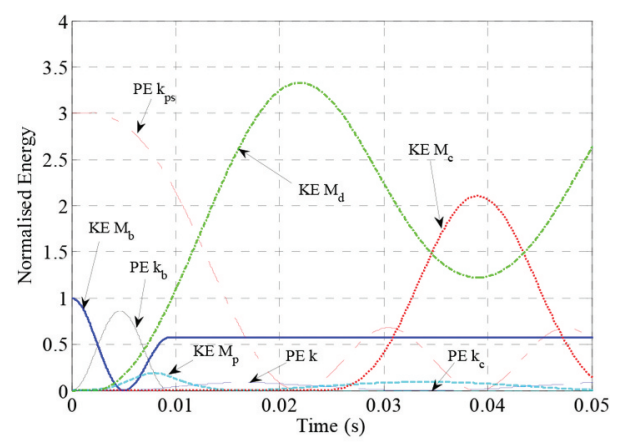

b. Energy fraction at point A

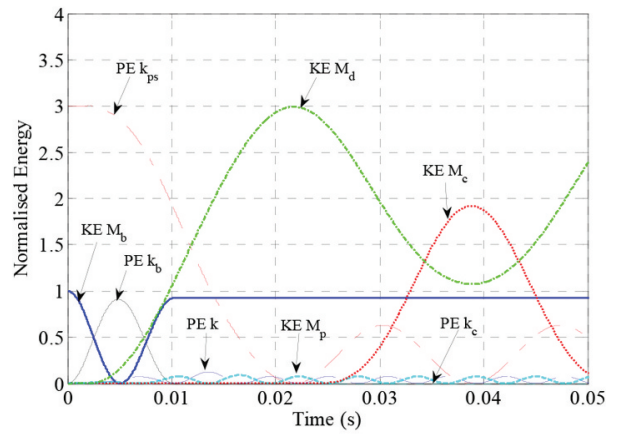

d. Energy fraction at point $\mathrm{C}$

Figure 7. Comparison of energy absorption ratio with the variation of $\omega_{p}$ 


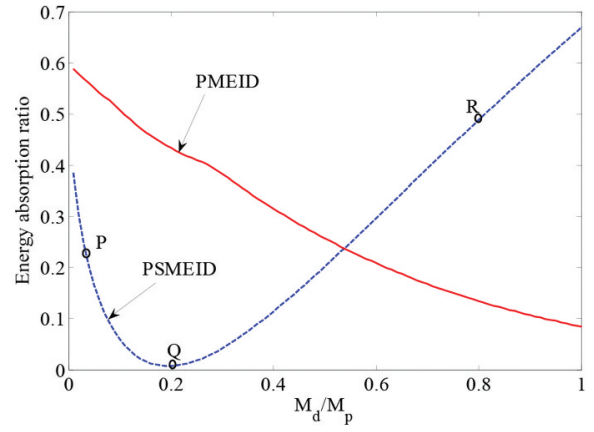

a. Energy absorption ratio vs. $M_{d}$

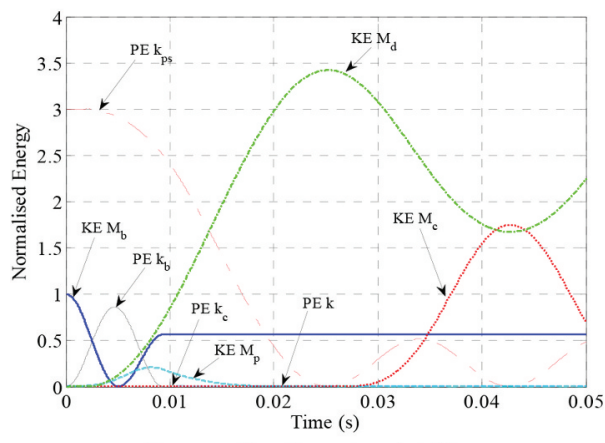

c. Energy fraction at point Q

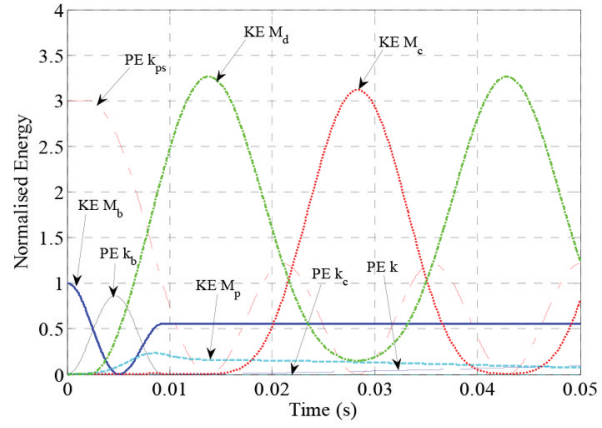

b. Energy fraction at point $\mathrm{P}$

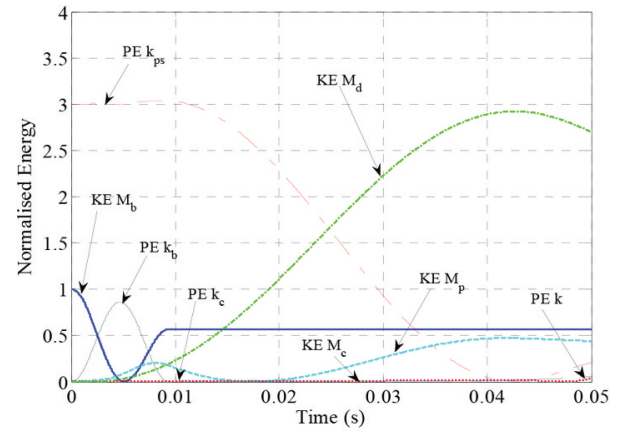

d. Energy fraction at point $\mathrm{R}$

Figure 8. Comparison of energy absorption ratio with the variation of $M_{d}$.

energy of the excitation mass. The small energy transfer is caused by a large damper mass inertia so that the response time becomes smaller.

Comparison of the acceleration and transmission force response for three cases, i.e., without a damper, with PMEID and PSMEID are shown in Figure 9. The acceleration and force transmission response obtained from cases without damper and with PMEID are not much different, as depicted in Figure 9. The transmission force is calculated from the deflection of the main spring $F_{\text {trans }}=k_{p} x_{p}$. The acceleration and force transmission response obtained using PSMEID is lower than those obtained using PMEID.

Figure 10 shows the primary mass displacement response for three cases, i.e., without a damper, with PMEID, and with PSMEID. The displacement response amplitude using PSMEID is much smaller

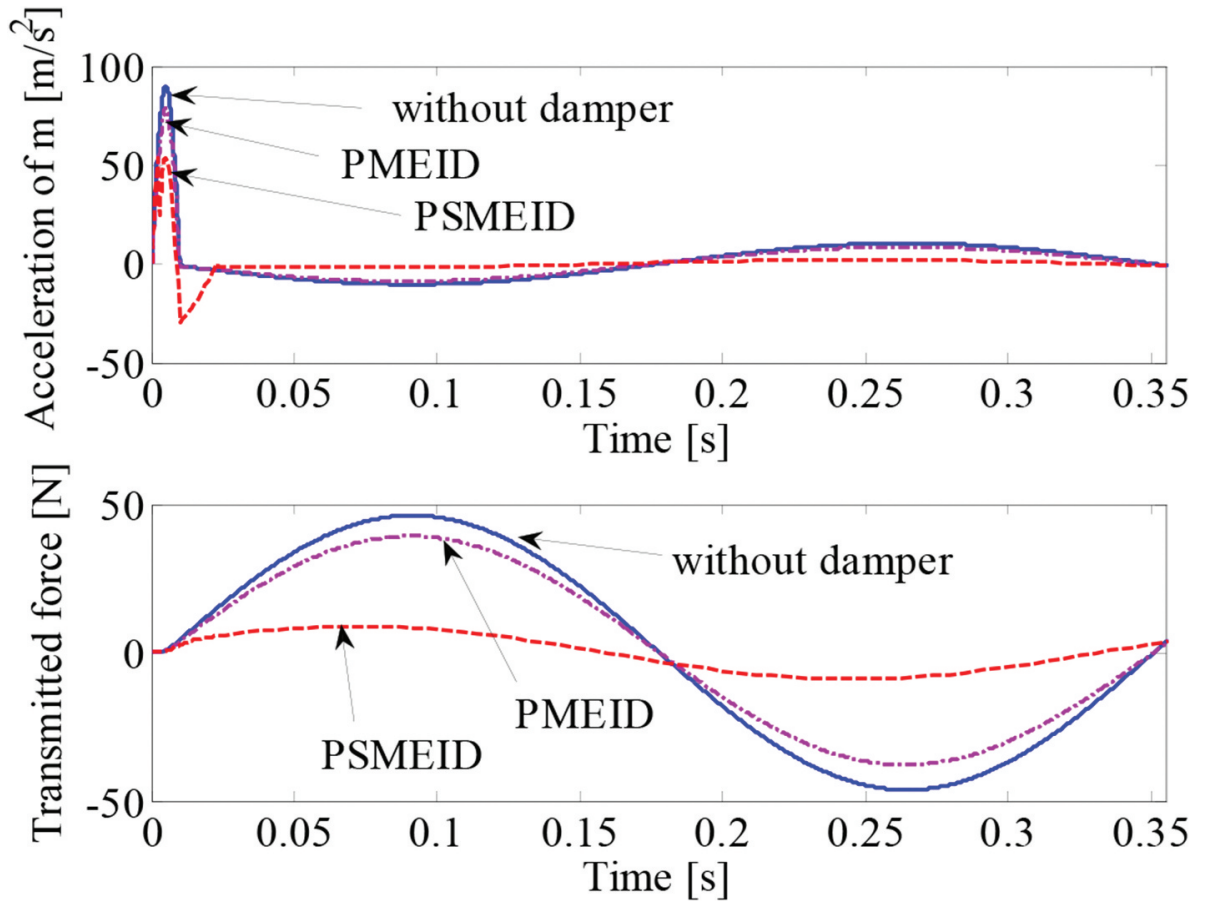

Figure 9. Acceleration and transmission force of primary mass. 


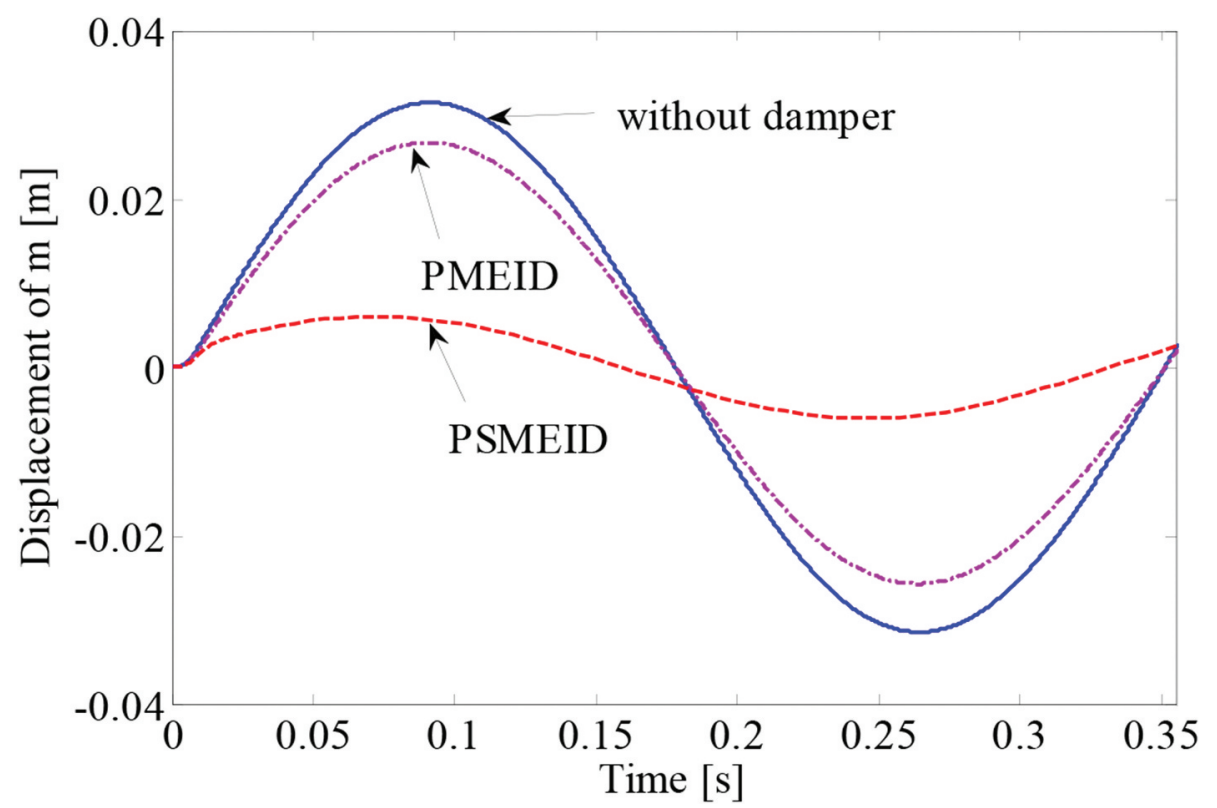

Figure 10. Displacement of primary mass.

in comparison with those obtained without damper and with PMEID. Base on the results obtained by a simulation study, it is shown that PSMEID not only attenuate the acceleration response but also reduce the displacement and force transmission.

\subsection{Experimental study}

The experimental apparatus of shock vibration control using PSMEID is shown schematically in Figure 11(a). The primary system is represented by a mass that is attached to the free end of a cantilever beam. The primary mass is a steel block with a mass of $4.5 \mathrm{~kg}$. The cantilever-mass system states one degree of freedom vibration model. The cantilever beam made of steel material. The beam length, width, and height are $800 \mathrm{~mm}, 35 \mathrm{~mm}$, and $7 \mathrm{~mm}$, respectively. The cantilever-mass system material and dimensions are selected that the first natural frequency of the system approaches the value used in the simulation. Contact condition between the primary mass $\left(M_{p}\right)$ and the contact mass $\left(M_{c}\right)$ is described using a contact spring. The acrylic resin material is used as the contact material between $M_{p}$ and $M_{c}$ to get the contact condition that is close to the simulation parameter. The PSMEID absorber consists of two masses, i.e., damper mass and contact mass, as depicted in Figure 11(b). The damper mass is an aluminium block with a mass of $0.67 \mathrm{~kg}$. A pre-straining $\operatorname{spring}\left(k_{p s}\right)$ is placed between the contact mass and the damper mass. An initial

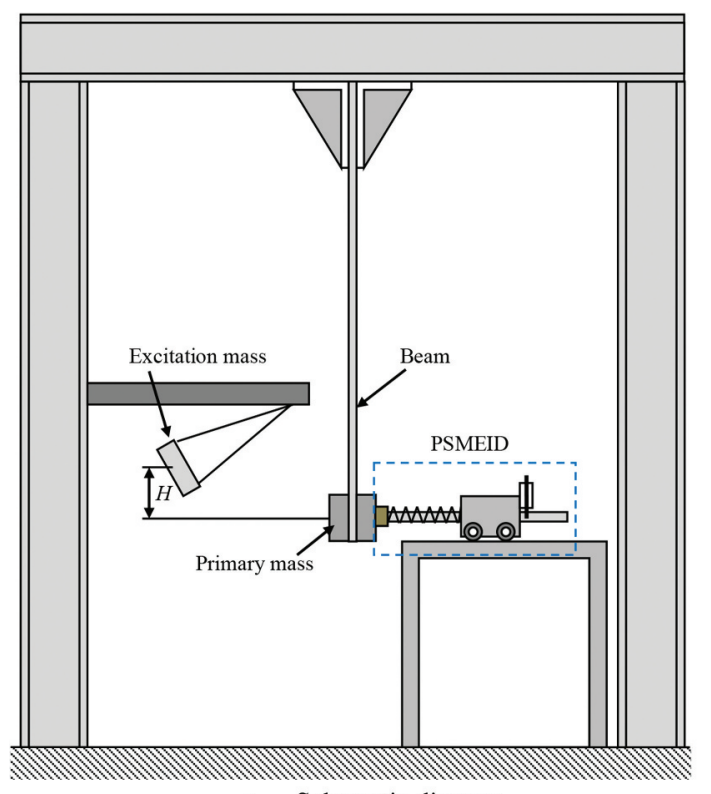

a. Schematic diagram

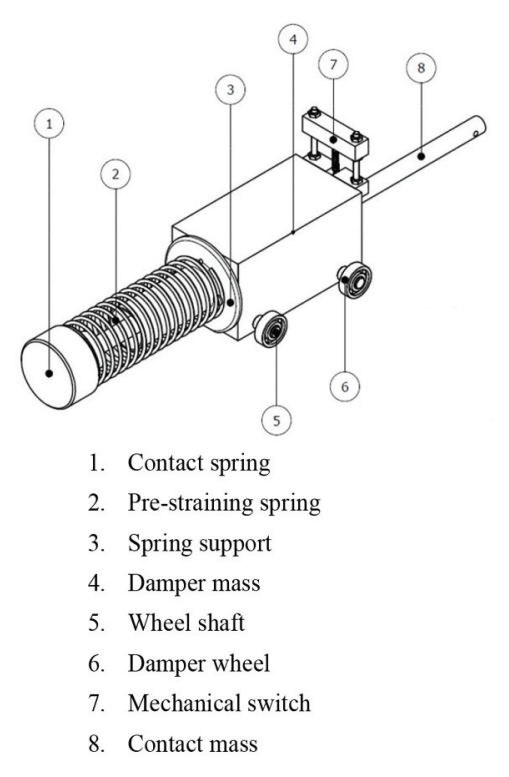

b. PSMEID mechanism

Figure 11. Experimental set-up. 
displacement is given to the pre-straining spring, and a mechanical switch is used to hold the pre-straining spring in its initial position. The mechanical switch consists of a cylindrical pin which is connected with a releasing spring. Immediately after the primary mass receives a shock load, the releasing spring pulls the pin-up. As a result, the pre-straining spring releases and simultaneously pushes the primary mass.

Impulsive excitation occurs due to a collision between the excitation mass $\left(M_{b}\right)$ and the primary mass $\left(M_{p}\right)$. In this case, the excitation mass is dropped freely from an initial height $(H)$, as shown in Figure 11 (a). A fall height of $15 \mathrm{~cm}$ is taken, which results in the same initial fall velocity as the simulation. The shock load intensity can be controlled by varying the initial dropping height of the excitation mass. To get the excitation frequency close to that used in the simulation, the contact surface between $M_{b}$ and $M_{p}$ is coated with a rubber sheet.

Figure 12 shows photographs of the experimental set-up. Numerical integration was implemented to obtain the displacement of the primary mass from its acceleration signal. The signal is recorded using the National Instrument USB-6221 BNC data acquisition system. The primary mass, contact mass, and impact damper mass are selected close to the value of the simulation parameters. The acceleration response of the primary mass is measured using an accelerometer located at the opposite side of the excitation point on the primary mass. The primary mass acceleration response is measured using a DeltaTron accelerometers type 4507 . The primary mass displacement is obtained from the integration process of the acceleration signal measured by the accelerometer.

Figure 13 shows the comparison of the primary mass acceleration response obtained by simulation and the experiment. Three conditions of response are evaluated, i.e., without a damper, with PMEID and PSMEID. Figure 13 shows that the maximum acceleration response using PSMEID is smaller than that of using PMEID. The largest impulsive response occurs after the impact. The impulsive signal obtained from the simulation study is perfectly a half sinusoidal function, but the experimental data is not perfectly a half sinusoidal signal. This result caused by the nonlinearity of the rubber contact stiffness between the excitation mass and the primary mass. The maximum acceleration peaks obtained from the simulation study almost similar to those obtained by the experimental study. The high-frequency signal occurs in the experimental data due to a high-frequency vibration component of the cantilever-mass system.

A comparison of the displacement response obtained from the simulation and the experiment is shown in Figure 14. The displacement response amplitude using PSMEID is lower than that of using PMEID. The displacement signal obtained from the simulation and the experimental study is almost similar. These results indicate that the experimental data can be used to validate the simulation results. Different from the acceleration response, the displacement response obtained by the experiment does not contain the high-frequency signal.

Figure 15 shows the maximum acceleration ratio of the primary mass with a variation of pre-straining

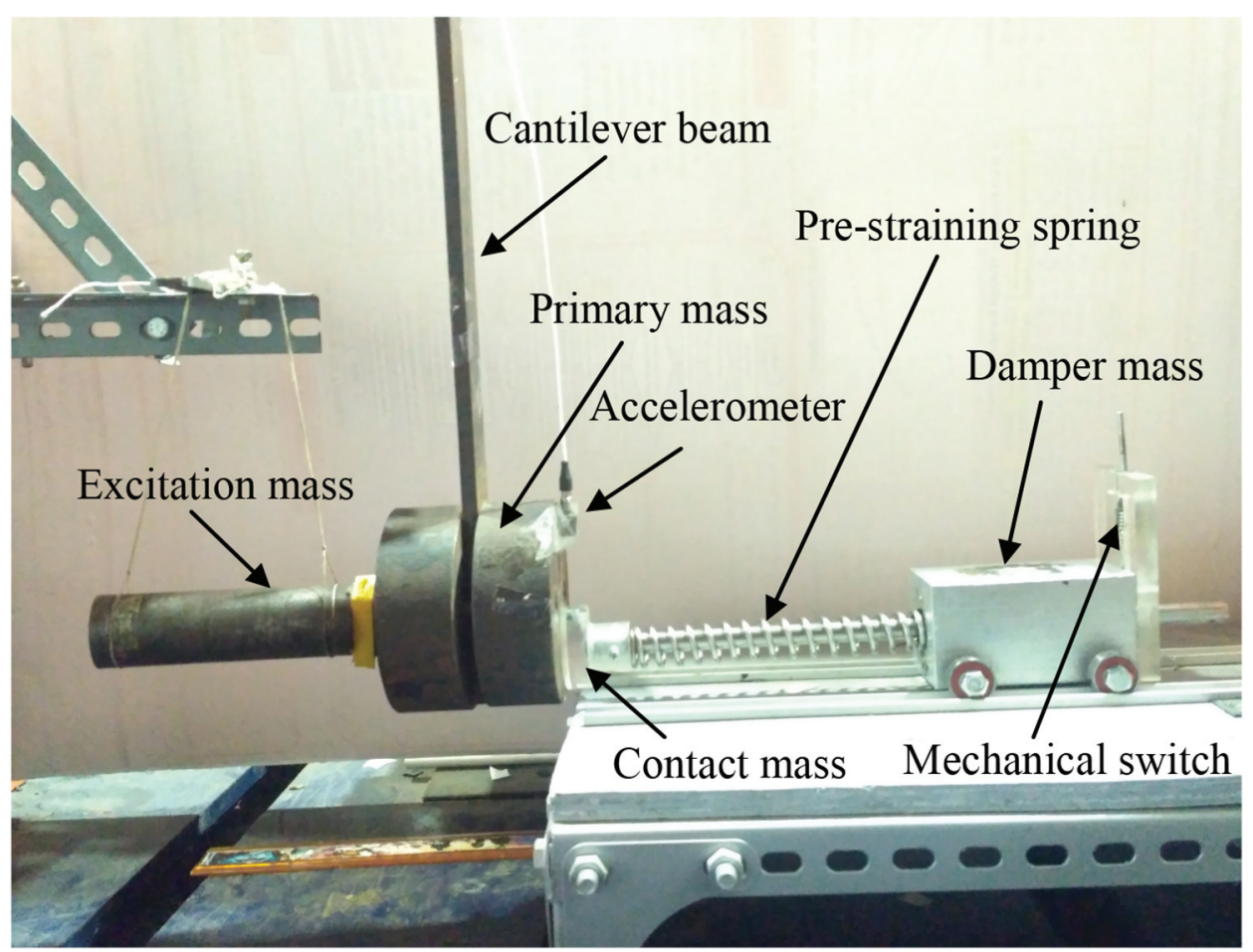

Figure 12. Photograph of the experimental set-up. 

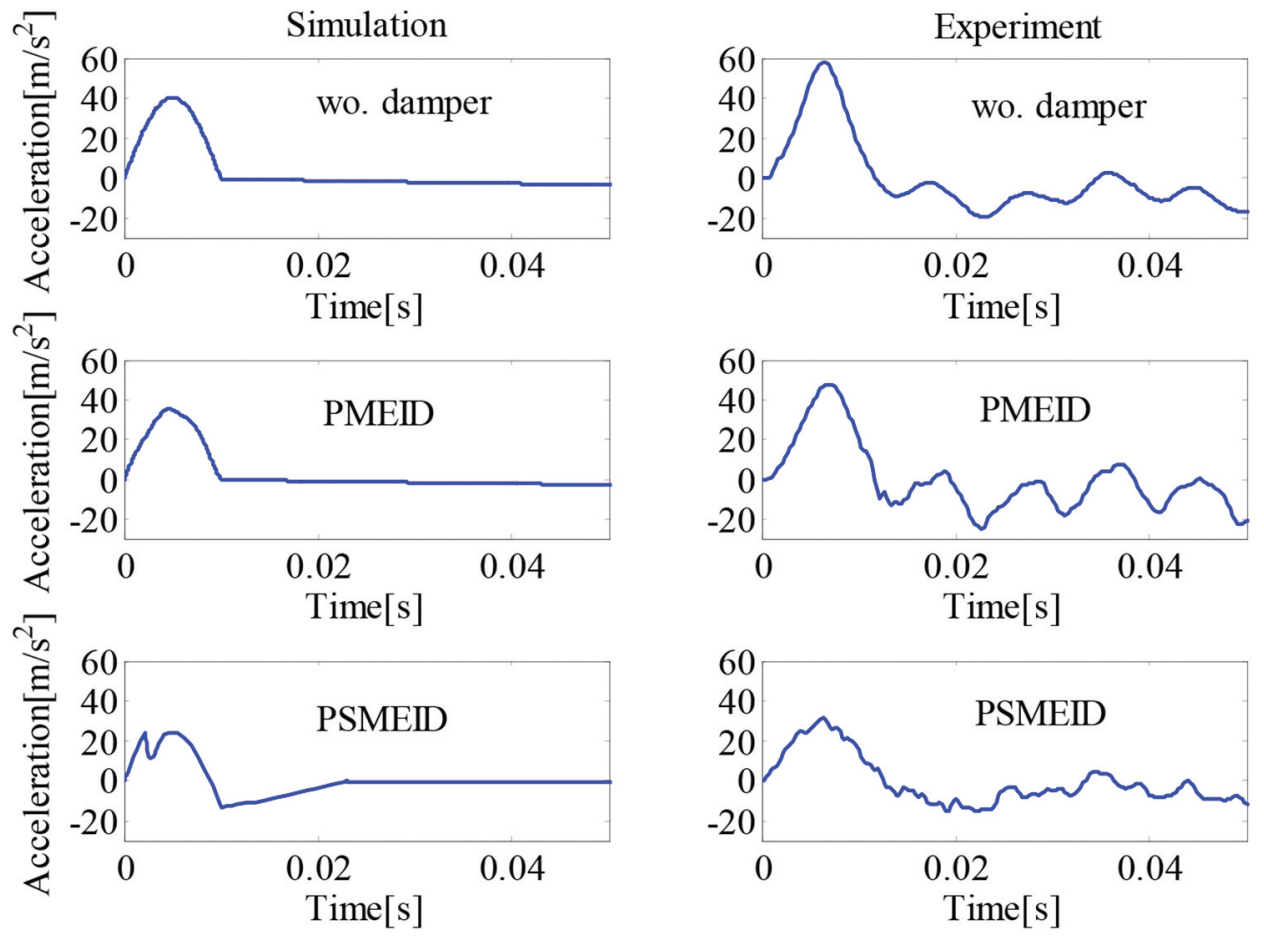

Figure 13. Comparison of the acceleration response.
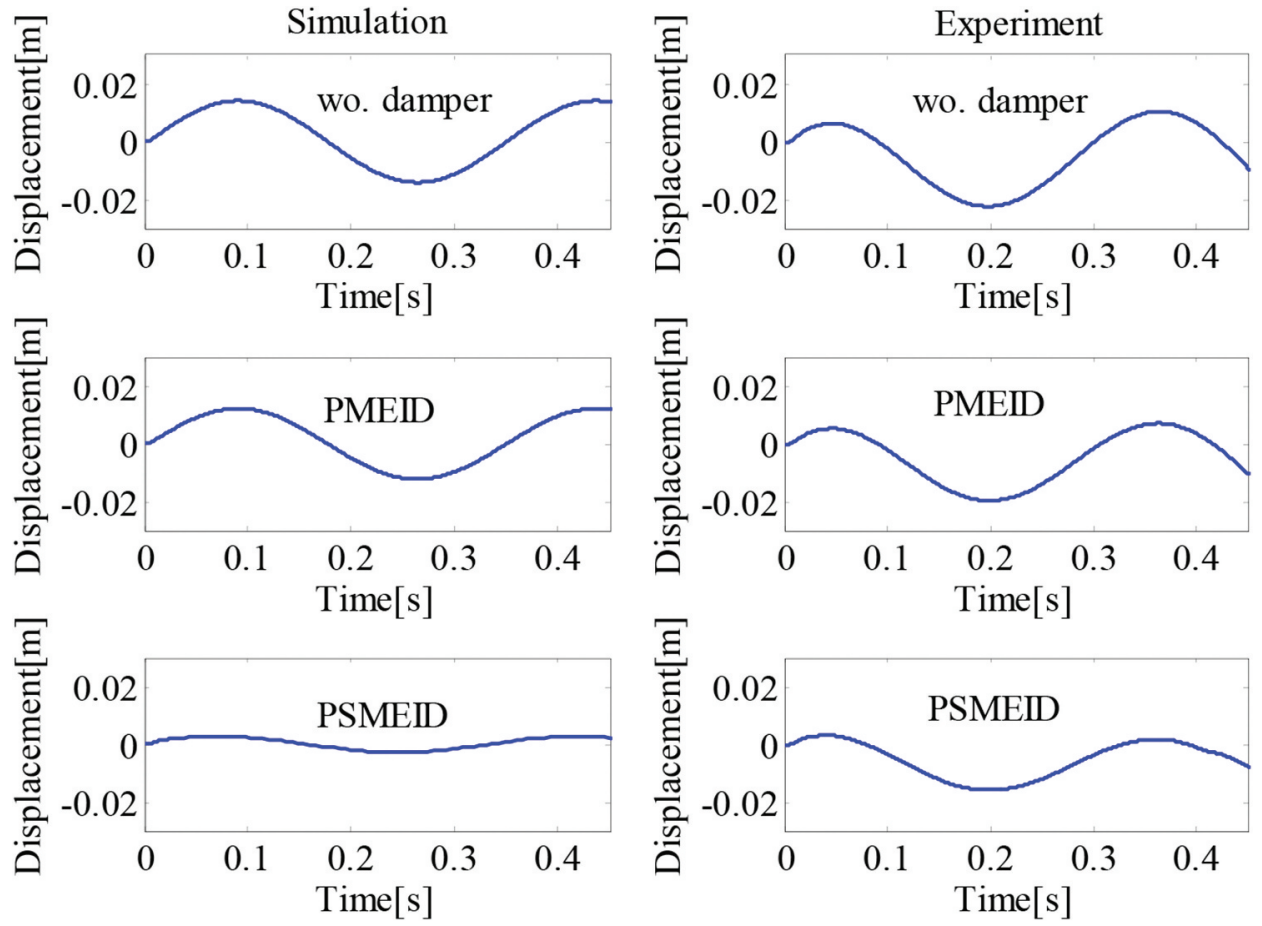

Figure 14. Comparison of the displacement response.

spring initial deflection. As depicted from Figure 15, the smallest value of the maximum acceleration occurs when $x_{p s} / x_{p s r e f}=0.18$. In this experimental study, the pin releasing force is kept constant. When $x_{p s} / x_{p s r e f}$ $=0$, the pre-straining spring is not compressed, and this spring acts as an additional contact stiffness to the Passive MEID system. Conversely, for $x_{p s} / x_{p s r e f}>0.18$, the pin doesn't release because the frictional force is large. For comparative study, four simulation results using several values of releasing pin force are also shown in Figure 15. As shown in Figure 15, for $f_{\text {pin }}$ If $f_{\text {ref }}=0.75$, the experimental results closest to that obtained by the simulation. The smallest acceleration ratio is obtained using $f_{\text {pin }} / f_{\text {fref }}=0.95$.

Even though the experimental data can validate the simulation results; however, due to complexities involved in the experiments, a number of parameters used in the experiment are slightly different with that 


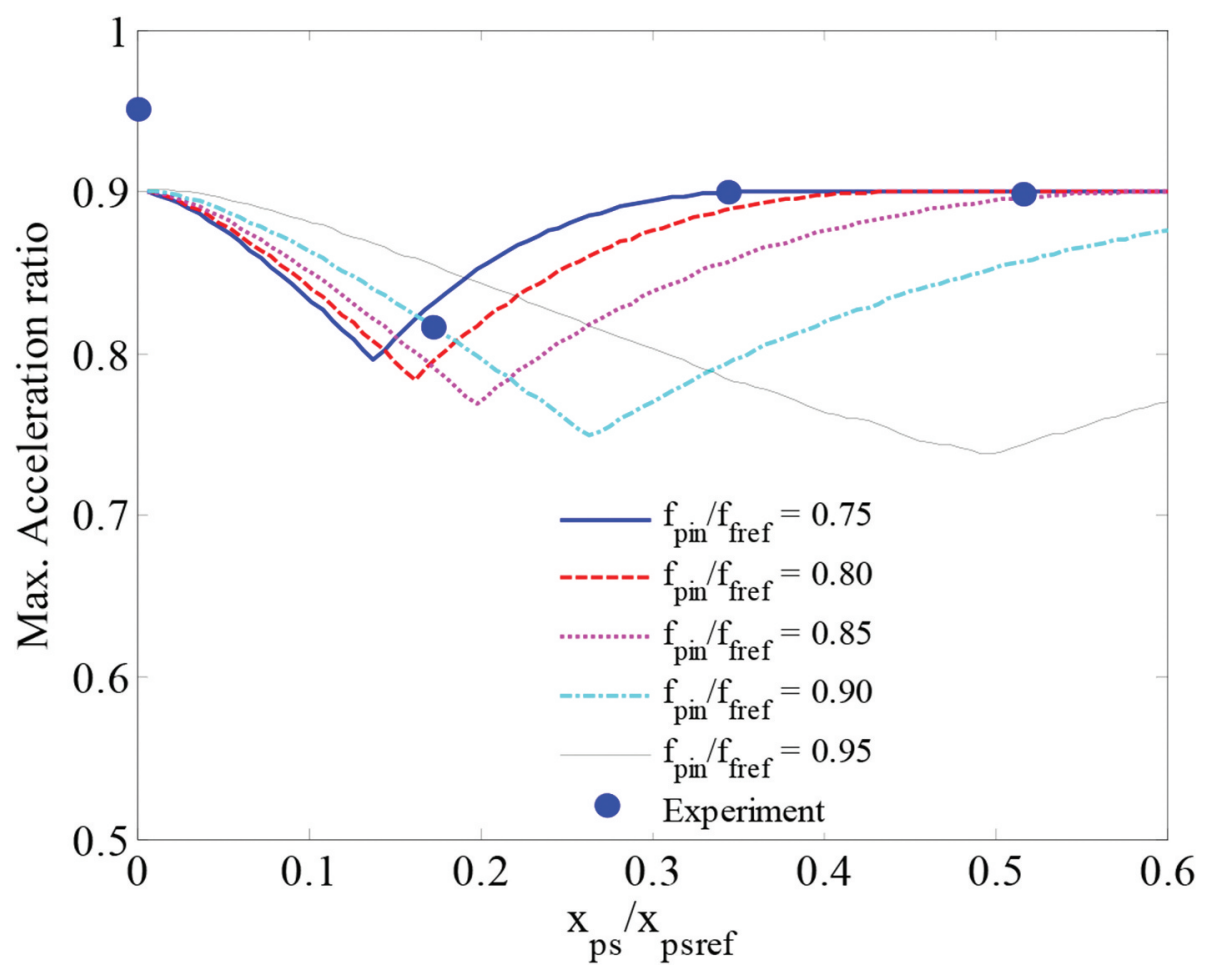

Figure 15. Maximum acceleration ratio vs $x_{p s}$

used in the simulation study. Based on the results obtained from the simulation study, PSMEID performance largely depends on the selection of the pin releasing force $\left(f_{\text {pin }}\right)$. The best performance is obtained if the pin force closest to the friction force. However, this condition is very rarely obtained in the experimental study because the real value of the friction force is unknown. This condition is resulting in some failed experimental data. As shown in Figure 15 , the difference between the experimental data and the simulation results is large enough, especially for a small value of pre-straining spring initial deflection $\left(x_{p s}\right)$. Another factor that causes different results between the experiment and simulation is the assumption of linear contact spring in the simulation study. This assumption is very difficult to realise in the experimental study because most of the contact condition has nonlinear characteristic.

\section{Conclusion}

Energy analysis and experimental evaluation of one degree of freedom system using PSMEID have been conducted. Several important results are summarised as follows:

(1) The fraction of energy absorbed by the primary mass was small if the excitation frequency differs from the primary mass natural frequency.

(2) When the excitation frequency is lower than the primary mass natural frequency, most of the impact energy is transferred to the damper mass, and for excitation frequencies higher than the primary mass natural frequency, a large number of the impact energy is reflected.

(3) The optimum condition of the pre-straining spring stiffness increases when the mass ratio is increased.

(4) The experimental study is conducted to validate the simulation results. It was shown from the experiment that the results have well agreed with those obtained from the simulation.

\section{Acknowledgments}

Financial support from the acceleration of professor research scheme No: 6/UN.16.17/PP.PGB/LPPM/2018, Andalas University year 2018, is gratefully acknowledged. I also thank to Mr. Defri and Mr. Nurmansyah who have lent their helping during preparation of the experimental set-up.

\section{Disclosure statement}

No potential conflict of interest was reported by the authors.

\section{Funding}

This work was supported by the Universitas Andalas [No: 6/ UN.16.17/PP.PGB/LPPM/2018].

\section{Notes on contributors}

Lovely Son, received a bachelor degree from the Mechanical Engineering Department, Faculty of Engineering, Andalas 
University, in 1998. He received a master degree from Mechanical Engineering Department, Bandung Institute of Technology, Indonesia, in 2000. He got doctoral degree from mechanical engineering Department, Kyoto University, Japan, in 2007. His current research is impact vibration attenuation and passive vibration control using dynamic vibration absorber.

Eka Satria, received bachelor degree from the mechanical engineering department, Andalas University, Indonesia, in 1999, and master degree from the mechanical engineering department, University of Leeds, England, in 2001. He got a doctor of engineering degree from Toyohashi University of Technology, Japan, in 2008. His current research is structural stability and optimization.

Jhon Malta, received his bachelor degree from the mechanical engineering department, Andalas University in 1999, and his Master degree in the mechanical engineering department, Bandung Institute of Technology, Indonesia, in 2001 and his doctoral degree from Technische Universitat Darmstadt, Germany, in 2009. His main fields of research were rotor dynamic and structural dynamic.

Berry Yuliandra, received bachelor degree from the Department of industrial engineering, Andalas University, in 2011. He received a Master degree from the Mechanical Engineering Department, Andalas University, in 2014. He currently works at the Department of Mechanical Engineering, Andalas University. He does research in Manufacturing System Engineering and industrial engineering.

\section{References}

Bovenzi, M., F. Rui, C. Negro, F. Agostin, G. Angotzi, S. Bianchi, L. Brahmati, et al. 2006. "An Epidemiological Study of Low Back Pain in Professional Drivers." Journal of Sound and Vibration. 298(3):514-539. doi:10.1016/j. jsv.2006.06.001

Constantinou, M., and M. D. Symans. 1993. "Seismic Response of Structure with Supplemental Damping." The Structural Design of Tall Building. 2(2):77-92. doi:10.1002/tal.4320020202

Dutta, D. 2020. "Semi-active Suspension System of Car Model Design: A Comparative Study." Australian Journal of Mechanical Engineering. 18(1):16-25. doi:10.1080/14484846.2017.1372029

Faraj, R., and C. Graczykowski. 2019. "Hybrid Prediction Control for Self-adaptive Fluid-based Shock-absorber." Journal of Sound and Vibration. 449:427-446. doi:10.1016/j.jsv.2019.02.022

Goel, R. K. 2005. "Seismic Response of Linear and Non-linear Asymmetric Systems with Nonlinear Viscous Dampers." Earthquake Engineering Structural Dynamics. 34(7):846-852. doi:10.1002/ eqe. 459
Goyda, H., and C. A. Teh. 1989. "A Study of the Impact Dynamics of Loosely Supported Heat Exchanger Tubes.” ASME Journal of Pressure Technology. 111(4):394-401. doi:10.1115/1.3265696

Hanagan, L. M., T. M. Murray, and K. Premaratne. 2003. "Controlling Floor Vibration with Active and Passive Devices." The Shock and Vibration Digest. 35 (5):347-365. doi:10.1177/05831024030355001

Lee, D. Y., Y. J. Nam, R. Yamane, and M. K. Park. 2009. "Performance Evaluation on Vibration Control of MR Landing Gear." Journal of Physics Conference Series. 149:012068.

Raju, K. R., and B. Annesh. 2017. "Analysis and Design of Foundation Systems to Control the Vibrations Due to Forging Impact Hammer." Journal of Structural Engineering. 44(5):404-413.

Rantaharju, T., N. J. Mansfield, H. J. M. Ala, and T. P. Gunston. 2015. "Predicting the Health Risks Related to Whole-body Vibration and Shock: A Comparison of Alternative Assessment Methods for High-acceleration Events in Vehicles." Ergonomics. 58 (7):1071-1087. doi:10.1080/00140139.2014.959071

Sezgin, A., and Y. Z. Arslan. 2012. "Analysis of the Vertical Vibration Effects on R Ide Comfort of Vehicle Driver.” Journal of Vibroengineering. 14(2):517-559.

Son, L., M. Bur, and M. Rusli. 2017. "Fundamental Study of Momentum Exchange Impact Damper Using Pre-straining Spring Mechanism." International Journal of Acoustic and Vibration. 22(4):422-430. doi:10.20855/ijav.2017.22.4487

Son, L., M. Bur, and M. Rusli. 2018. "A New Concept for UAV Landing Gear Shock Vibration Control Using Pre-straining Spring Momentum Exchange Impact Damper." Journal of Vibration and Control. 24 (8):1455-1468. doi:10.1177/1077546316661470

Son, L., S. Hara, K. Yamada, and H. Matsuhisa. 2010. "Experiment of Shock Vibration Control Using Active Momentum Exchange Impact Damper." Journal of Vibration and Control. 16(1):49-64. doi:10.1177/ 1077546309102675

Son, L., M. Kawachi, H. Matsuhisa, and H. Utsuno. 2007. "Reducing Floor Impact Vibration and Sound Using a Momentum Exchange Impact Damper." Journal of System Design and Dynamics. 1(1):14-26. doi:10.1299/ jsdd.1.14

Son, L., H. Matsuhisa, and H. Utsuno. 2008. "Energy Transfer in a Three Body Momentum Exchange Impact Damper." Journal of System Design and Dynamics. 2 (1):425-441. doi:10.1299/jsdd.2.425

Tung, P. C., and S. W. Shaw. 1998. "The Dynamic of an Impact Print Hammer." Journal of Vibration, Acoustic, Stress, and Reliability in Design. 110(2):193-200. doi:10.1115/1.3269498

Yang, Y., and X. Wang. 2019. "Investigation into the Linear Velocity Response of Cantilever Beam Embedded with Impact Damper." Journal of Vibration and Control. 25(7):1365-1378. doi:10.1177/ 1077546318821711 